TRANSACTIONS OF THE

AMERICAN MATHEMATICAL SOCIETY

Volume 355, Number 9, Pages 3625-3639

S 0002-9947(03)03259-8

Article electronically published on May 29, 2003

\title{
THE COMBINATORIAL RIGIDITY CONJECTURE IS FALSE FOR CUBIC POLYNOMIALS
}

\author{
CHRISTIAN HENRIKSEN
}

\begin{abstract}
We show that there exist two cubic polynomials with connected Julia sets which are combinatorially equivalent but not topologically conjugate on their Julia sets. This disproves a conjecture by McMullen from 1995 .
\end{abstract}

\section{INTRODUCTION AND RESULT}

Let $\mathcal{P}_{d}=\left\{z^{d}+a_{d-2} z^{d-2}+\cdots+a_{0}\right\} \leftrightarrow \mathbb{C}^{d-1}$ be the space of monic centered polynomials of degree $d>1$.

Our object is to show that there exists a cubic polynomial $f \in \mathcal{P}_{3}$ that is not combinatorially rigid. To specify what this means, we need to introduce some notation and results from the theory of holomorphic dynamics, and, more specifically, the dynamics of polynomials. We will assume that the reader has some knowledge of the theory of iteration of polynomials in one variable. There are several introductory books on holomorphic dynamics, such as [M1]; also Lyubich [L1] has recently published a survey article.

The filled Julia set $K(f)$ of $f \in \mathcal{P}_{d}$ is the set of values of $z$ such that the orbit $z, f(z), f \circ f(z)=f^{2}(z), \ldots$ is bounded. The boundary of this set $J(f)=\partial K(f)$ is called the Julia set of $f$, and it coincides with the set of points that admit no neighborhood restricted to which the iterates id, $f, f^{2}, \ldots$ form a normal family.

We denote by $\mathcal{C}_{d}$ the connectedness locus of the degree $d$ monic centered polynomials:

$$
\mathcal{C}_{d}=\left\{f \in \mathcal{P}_{d} \mid K(f) \text { is connected }\right\}
$$

The connectedness locus of the quadratic polynomials, $\mathcal{C}_{2}$, is the Mandelbrot set, and we also denoted it by $M$.

Given $f \in \mathcal{C}_{d}$, there exists a unique conformal isomorphism

$$
\phi_{f}: \mathbb{C} \backslash K(f) \stackrel{\simeq}{\rightarrow} \mathbb{C} \backslash \overline{\mathbb{D}}
$$

that conjugates $f$ to $z \mapsto z^{d}$ and satisfies $\phi_{f}(z)=z+\mathcal{O}(1 / z)$. The conformal isomorphism is called a Böttcher coordinate.

Using the Böttcher coordinate, we can define the notion of the dynamical ray $R_{f}(\theta)$ of angle $\theta \in \mathbb{R} / \mathbb{Z}$ as the preimage $\phi_{f}^{-1}\{\exp (\eta+2 i \pi \theta) \mid \eta>0\}$ of a radial segment. For a polynomial $f \in \mathcal{P}_{d}$, we define the potential $G_{f}: \mathbb{C} \backslash K(f) \rightarrow \mathbb{R}$ by

$$
G_{f}(z)=\lim _{n \rightarrow+\infty} \frac{1}{d^{n}} \log \left|f^{n}(z)\right| \text {. }
$$

Received by the editors January 30, 2002 and, in revised form, August 13, 2002.

2000 Mathematics Subject Classification. Primary 37F10; Secondary 37F20, 37F45.

This research was funded by a Marie Curie Fellowship. 
Then, when $K(f)$ is connected, $G_{f}=\log \left|\phi_{f}\right|$, and a dynamical ray is parallel to the vector field grad $G_{f}$. This allows us to generalize the notion of a dynamical ray. Indeed, for an arbitrary polynomial $f \in \mathcal{P}_{d}$, the dynamical ray $R_{f}(\theta)$ is the curve that is tangent to grad $G_{f}$ and tangent to the segment $\{r \exp (2 i \pi \theta) \mid r>0\}$ near infinity.

Following a dynamical ray down potential, two things might happen. Either the ray hits a critical point of $G_{f}$ and we say it bifurcates, or it accumulates on the Julia set. The first behaviour only takes place when $G_{f}$ has critical points, which is equivalent to $f$ having critical points that are attracted to infinity and to $K_{f}$ being disconnected.

Suppose the ray $R_{f}(\theta)$ does not bifurcate. Consider $\overline{R_{f}(\theta)} \backslash R_{f}(\theta)$. This continuum is called the accumulation set of the ray. If it consists of only one point, we say that the ray lands. In the connected case, $f \in \mathcal{C}_{d}$, that means that the limit $z_{0}=\lim _{\eta \backslash 0} \phi_{f}-1 \circ \exp (\eta+2 i \pi \theta)$ exists.

Rays of rational angle are special, in that they always land when $f \in \mathcal{C}_{d}$ :

Proposition 1. Let $f \in \mathcal{P}_{d}$ and $\theta \in \mathbb{Q} / \mathbb{Z}$. Then the ray $R_{f}(\theta)$ either bifurcates or lands at a (pre)periodic point $\gamma$. In the latter case, $\gamma$ is periodic if $\theta$ is periodic, and preperiodic if $\theta$ is preperiodic; it is either repelling or parabolic.

The proposition is proved in the Orsay Notes (Proposition 2 of exposé 8 in DH1).

This proposition allows us to distinguish the two fixed points of a quadratic polynomial $P_{c}: z \mapsto z^{2}+c$ when $c \in M \backslash\{1 / 4\}$ (for $c=1 / 4, P_{c}$ has one fixed point and it is of multiplicity 2$)$. Indeed, the ray $R_{P_{c}}(0)$ is fixed by $P_{c}$ and then must land at a fixed point. This fixed point is called the $\beta$-fixed point, and the other fixed point is called the $\alpha$-fixed point.

Definition 1. Following McMullen ([Mc1] $)$ we define the rational lamination $\lambda_{\mathbb{Q}}(f)$ $\subset \mathbb{Q} / \mathbb{Z} \times \mathbb{Q} / \mathbb{Z}$ of $f \in \mathcal{C}_{d}$ to be the equivalence relation under which two rational angles $\theta^{\prime}$ and $\theta^{\prime \prime}$ are equivalent if and only if the two dynamical rays $R_{f}\left(\theta^{\prime}\right)$ and $R_{f}\left(\theta^{\prime \prime}\right)$ land at the same point.

Definition 2. A polynomial $f \in \mathcal{C}_{d}$ with no indifferent cycles is said to be combinatorially rigid if for every $g \in \mathcal{C}_{d}$ with no indifferent cycles such that $\lambda_{\mathbb{Q}}(f)=\lambda_{\mathbb{Q}}(g)$, the composition of Böttcher coordinates $\phi_{g}^{-1} \circ \phi_{f}$ extends to a quasiconformal homeomorphism on the whole Riemann sphere.

Note that we do not require the extension to be a conjugacy on the sphere; however, by continuity, it will be a conjugacy on the Julia set $J(f)$.

The following statement has been conjectured:

Every polynomial $f \in \mathcal{C}_{d}$ without indifferent periodic cycles is combinatorially rigid. (See [Mc1.)

This conjecture can be viewed as a series of conjectures, one for each value of $d=2,3, \ldots$

If the conjecture is true for quadratic polynomials $(d=2)$, then it would imply that the Mandelbrot set $M$ is locally connected [Sch]. Local connectivity of $M$ is one of the most important questions in the study of the dynamics of quadratic polynomials. It has been verified for most types of parameters in the boundary of $M$. A summary of known results on this question is given by Lyubich in [L2], Appendix B. 
We shall show that the conjecture is false in degree 3, by finding two cubic polynomials with connected Julia sets, with no indifferent cycles and the same rational lamination, but which fail to be even topologically conjugate on their Julia sets.

A sketch of the proof follows: From work of Sørensen $[\mathrm{S} \varnothing]$ it follows that there is an infinitely renormalizable quadratic polynomial $P_{c_{\infty}}: z \mapsto z^{2}+c_{\infty}$ whose Julia set contains a subcontinuum without periodic or preperiodic points. Choose a quadratic polynomial $Q$ whose critical point eventually lands at the $\beta$-fixed point. Let $f_{0}$ be the result of intertwining $Q$ with $P_{c_{\infty}}$ at their $\beta$-fixed points, using the intertwining surgery construction of Epstein and Yampolsky [EY].

The cubic polynomial $f_{0}$ has a quadratic-like restriction $f_{0}: U^{\prime} \rightarrow U$ that is hybrid equivalent to $P_{c_{\infty}}$.

There is a neighborhood $\Lambda$ of $f_{0}$ such that for each $f_{\lambda}$ there are disks $U_{\lambda}^{\prime}, U_{\lambda}$ for which the restriction $f_{\lambda}: U_{\lambda}^{\prime} \rightarrow U_{\lambda}$ is quadratic-like. This can be accomplished so that the family $\left(f_{\lambda}: U_{\lambda}^{\prime} \rightarrow U_{\lambda}\right)$ is an analytic family in the sense of [DH2]. Using results in $\mathrm{DH} 2$, we can suppose that the set of parameters for which the corresponding quadratic-like mappings all are hybrid equivalent to $P_{c_{\infty}}$ locally forms a codimension-one submanifold of the space of cubic polynomials.

For parameters $t$ in this submanifold, one critical point $\omega_{1}(t)$ belongs to this quadratic-like restriction, whereas the other $\omega_{2}(t)$ does not. Using holomorphic motion arguments, we see that there are copies of (part of) the Julia set of $P_{c_{\infty}}$ showing up in the set of $t$-values for which $f_{t}$ has connected Julia set. Loosely speaking, the position of the parameter $t$ in such a parameter copy corresponds to the position of an iterate of $\omega_{2}(t)$ in a copy of $K\left(P_{c_{\infty}}\right)$, in the dynamical plane. So by varying $t$ we can slide an iterate of $\omega_{2}(t)$ around in a continuum without periodic or preperiodic points. It follows that no rational ray bifurcates. However, we shall see that the dynamics on the Julia sets do change.

\section{A sPeCial QUADRATiC POLYNOMial}

In this section we will find a quadratic polynomial that does not have indifferent cycles and whose Julia set contains a continuum with no periodic or preperiodic cycles.

\section{Polynomial-like mappings and renormalization.}

Definition 3. If $U^{\prime}, U$ are topological disks with $U^{\prime}$ compactly contained in $U$ and $f: U^{\prime} \rightarrow U$ is a holomorphic, ramified covering of degree $d>1$, then $f: U^{\prime} \rightarrow U$ is called a polynomial-like map of degree $d$.

The notion of polynomial-like mappings was introduced by Douady and Hubbard (see $[\mathrm{DH} 2$ ) and is extremely useful. When the degree of a polynomial-like map is two, we call it quadratic-like. As the name suggests, a quadratic-like map behaves qualitatively like a quadratic polynomial.

Analogously to the definitions for polynomials, we define the filled Julia set of a polynomial-like map $f$ as the set of points that do not escape under iteration: $K(f)=\left\{z \in U^{\prime} \mid f^{1}(z) \in U^{\prime}, f^{2}(z) \in U^{\prime}, \ldots\right\}$, and the Julia set $J(f)$ of $f$ as its boundary: $J(f)=\partial K(f)$. Two polynomial-like mappings $f, g$ are called hybrid equivalent if there exists a quasiconformal mapping $\phi$ that maps a neighborhood of $K(f)$ onto a neighborhood of $K(g)$, conjugating $f$ to $g$ and satisfying $\frac{\bar{\partial} \phi}{\partial \phi}=\frac{\partial \phi / \partial \bar{z}}{\partial \phi / \partial z}=$ 
0 almost everywhere on $K(f)$. This clearly defines an equivalence relation, whose equivalence classes are called hybrid classes.

That quadratic-like mappings behave like quadratic polynomials follows from Douady and Hubbard's Straightening Theorem, which for quadratic-like mappings boils down to:

Straightening Theorem. A quadratic-like map $f$ is hybrid equivalent to a quadratic polynomial. If $K(f)$ is connected, $f$ is hybrid equivalent to a unique monic centered polynomial $z \mapsto z^{2}+\chi(f)$.

It may happen that an iterate of a quadratic polynomial in some region behaves like a quadratic-like map.

Definition 4. A quadratic polynomial $P: z \mapsto z^{2}+c$ is called n-renormalizable for an integer $n>1$, if there exists a restriction $P^{n}: U^{\prime} \rightarrow U$ that is quadraticlike with connected Julia set and $0 \in U^{\prime}$. The restriction $P^{n}: U^{\prime} \rightarrow U$ is called a renormalization of $P^{n}$, and the set of positive integers $n>1$ such that $P$ is $n$-renormalizable is called the levels of renormalization of $P$ and is denoted $\mathcal{R}(P)$. If this set is nonempty, $P$ is called renormalizable, and if it is infinite, $P$ is called infinitely renormalizable.

In [Mc2] McMullen shows:

Theorem 2 (Uniqueness of renormalization). Any two renormalizations of $P^{n}$ have the same filled Julia set.

An infinitely renormalizable quadratic polynomial. If $n \in \mathcal{R}(P)$, we denote by $K_{n}(P)$ the uniquely determined filled Julia set of $P^{n}: U^{\prime} \rightarrow U$, and we set $J_{n}(P)=\partial K_{n}(P)$.

In $[\mathrm{S} \varnothing]$, Sørensen proves the following result:

Theorem 3. There is an infinitely renormalizable quadratic polynomial $P_{c_{\infty}}: z \mapsto$ $z^{2}+c_{\infty}$ with the following properties: There is a sequence of integers $n_{1}<n_{2}<\cdots$ such that each $n_{k} \in \mathcal{R}\left(P_{c_{\infty}}\right), K_{n_{k}} \supset K_{n_{k+1}}$ and the continuum $J_{\infty}=\bigcap K_{n_{k}}\left(P_{c_{\infty}}\right)$ is nontrivial (i.e., contains more than one point).

A proof can also be found in $1 \mathrm{M} 2$.

Let $P_{c_{\infty}}$ and $J_{\infty}$ be as given by Theorem 3 Since $J_{\infty}$ is obtained as an intersection of nested full continua, $J_{\infty}$ is a full continuum.

By the following theorem of McMullen, $J_{\infty}$ contains no periodic or preperiodic points.

Theorem 4. If a quadratic polynomial $P$ is infinitely renormalizable, then:

(1) all periodic cycles of $P$ are repelling;

(2) the filled Julia set $K(P)$ has no interior; and

(3) if $R \subset \mathcal{R}(P)$ is infinite, then the continuum $\bigcap_{n \in R} K_{n}(P)$ contains no periodic or preperiodic points.

Proof. This is an immediate consequence of [Mc2], Theorems 8.1 and 7.8.

Arbitrarily small copies of $J_{\infty}$ accumulate on the $\beta$-fixed point of $P_{c_{\infty}}$ :

Proposition 5. Given any $\epsilon>0$, there are an integer $n>0$ and a topological disk contained in $\{|z-\beta|<\epsilon\}$ that $P_{c_{\infty}}^{n}$ maps univalently onto a neighborhood of $J_{\infty}$. 


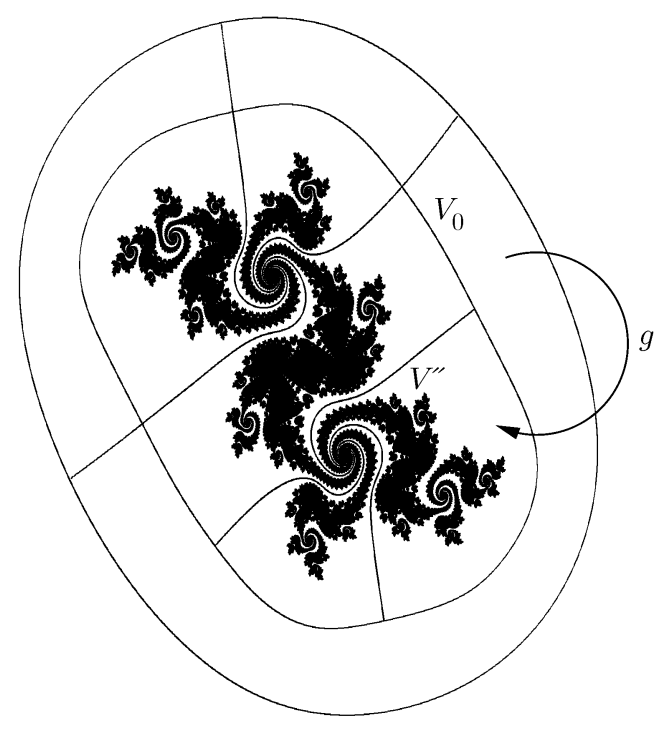

Figure 1. The inverse branch $g$ used in the proof of Proposition 5 Due to inherent difficulties in drawing the filled Julia set $K\left(P_{c_{\infty}}\right)$, a filled Julia set for another parameter is drawn.

Proof. By Theorem 4 the $\alpha$-fixed point, $\alpha$, of $P_{c_{\infty}}$ is repelling, since $P_{c_{\infty}}$ is infinitely renormalizable. By a result of Douady and Yoccoz, $k$ rational rays land at $\alpha$ for some $k>0$. The ray of angle $0^{\circ}$ lands at the $\beta$-fixed point, $\beta$, and it follows that $k>1$. The region $W=\left\{z \mid G_{P_{c_{\infty}}}<1\right\}$ is a Jordan domain. The $k$ rational rays landing at $\alpha$ cut this region into $k$ pieces $V_{0}, V_{1}, \ldots, V_{k-1}$, which we enumerate cyclically with respect to $\alpha$ in such a way that the critical point 0 is contained in $V_{0}$. The preimage $W^{\prime}$ of $W$ is cut into $2 k-1$ regions $V_{0}^{\prime}, V_{1}^{\prime}, \ldots, V_{2 k-2}^{\prime}$ by the rational rays landing at $\alpha$ and $-\alpha$. We can assume $0 \in V_{0}^{\prime}$. Each piece $V_{i}^{\prime}, i \neq 0$, is mapped properly of degree one to a piece $V_{\sigma(i)}$, and $V_{0}^{\prime}$ is mapped properly of degree 2 to a piece $V_{\sigma(0)}$. Since none of the rational rays landing at $\alpha$ are fixed, $V_{\sigma(0)}$ does not coincide with $V_{0}$. It follows that $V_{0}$ has two preimages: one $V^{\prime \prime}$ that is compactly contained in $V_{0}$, and the symmetric one $-V^{\prime \prime}$. Since 0 is contained in $J_{\infty}$ and $J_{\infty}$ contains neither $\alpha$ nor $-\alpha$, we get that $J_{\infty}$ is contained in $V_{0}$ and does not intersect $V^{\prime \prime}$.

Now consider the inverse branch $g=P_{c_{\infty}}^{-1}: V_{0} \rightarrow V^{\prime \prime}$ (see Figure 1). This univalent map is a strong contraction with respect to the hyperbolic metric on $V_{0}$ and necessarily contains a fixed point, which then must be $\beta$. Now $V_{0}, g\left(V_{0}\right), g^{2}\left(V_{0}\right), \ldots$ is a sequence of topological disks, each containing $\beta$ in its closure, whose diameters tend towards zero. Finally, $P_{c_{\infty}}^{n}$ maps $g^{n}\left(V_{0}\right)$ univalently onto $V_{0}$.

\section{Cubic polynomials}

In this section we consider the space of cubic polynomials, and see that deformed copies of (parts of) quadratic Julia sets show up both in dynamical planes and in parameter slices. 
We parametrize the space of cubic monic centered polynomials by $\mathbb{C}^{2}$, letting $f_{\mathbf{a}}(z)=z^{3}-3 a^{2} z+b, \mathbf{a}=(a, b) \in \mathbb{C}^{2}$. This is a four-to-one covering of the cubic polynomials modulo affine conjugacy, ramified over $\{a b=0\}$. The two critical points $\omega_{1}=\omega_{1}(\mathbf{a})=a$ and $\omega_{2}=\omega_{2}(\mathbf{a})=-a$ are then global holomorphic functions of the parameter $\mathbf{a}$.

Define $\mathcal{Q}_{1} \subset \mathbb{C}^{2}$ to be the set of parameters for which $f_{\text {a }}$ can be restricted to a quadratic-like mapping $f_{\mathbf{a}}: U^{\prime} \rightarrow U$ with $\omega_{1} \in U^{\prime}$. We say that $\mathcal{Q}_{1}$ is the set of parameters such that $f_{\mathbf{a}}$ is $\omega_{1}$-renormalizable, and we call $f_{\mathbf{a}}: U^{\prime} \rightarrow U$ an $\omega_{1}$-renormalization of $f_{\mathbf{a}}$. Similarly, denote by $\mathcal{Q}_{2}$ the set of parameters for which $f_{\mathbf{a}}$ is $\omega_{2}$-renormalizable. Notice that a cubic polynomial being $\omega_{i}$-renormalizable is quite different than a quadratic polynomial being renormalizable, since in the former case we are only considering restrictions of the cubic map, whereas in the latter case we only consider restrictions of some $n$th iterate, $n>1$. (Also, this terminology is not completely standard, in the sense that it is usually required that the filled Julia set of the renormalization be connected for a mapping to be called renormalizable. However, in this context we find it convenient to drop that requirement for $\omega_{i}$-renormalizations.)

An $\omega_{1}$-renormalization (or an $\omega_{2}$-renormalization) of $f_{\mathbf{a}}$ has the following properties, independent of the choice of domains:

Proposition 6. Suppose $f_{\mathbf{a}_{0}}$ is $\omega_{1}$-renormalizable, and let $f_{\mathbf{a}_{0}}: U_{1}^{\prime} \rightarrow U_{1}$ and $f_{\mathbf{a}_{0}}$ : $U_{2}^{\prime} \rightarrow U_{2}$ denote two $\omega_{1}$-renormalizations. Then $K\left(f_{\mathbf{a}_{0}}: U_{1}^{\prime} \rightarrow U_{1}\right)=K\left(f_{\mathbf{a}_{0}}: U_{2}^{\prime} \rightarrow\right.$ $\left.U_{2}\right)$. Furthermore, if $K\left(f_{\mathbf{a}_{0}}: U_{1}^{\prime} \rightarrow U_{1}\right)$ is connected, then both renormalizations are hybrid equivalent to the same quadratic polynomial $z \mapsto z^{2}+\chi_{1}\left(\mathbf{a}_{0}\right)$.

Proof. The result follows readily from [Mc2] (Theorem 5.11) and from the Straightening Theorem.

A one-dimensional submanifold of the cubic polynomials. Let $\mathcal{K}_{i} \subset \mathcal{Q}_{i}$, $i=1,2$, denote the subset of parameters a such that an $\omega_{i}$-renormalization of $f_{\mathbf{a}}$ has connected Julia set.

It follows from Proposition 6 that for a given point $\mathbf{a} \in \mathcal{K}_{i}$ there is a unique monic centered polynomial $z \mapsto z^{2}+\chi_{i}(\mathbf{a})$ that is hybrid-equivalent to an $\omega_{i^{-}}$ renormalization of $f_{\mathbf{a}}$. This determines mappings $\chi_{i}$ of $\mathcal{K}_{i}$ into the Mandelbrot set $M$.

Theorem 7. There exists a parameter $\mathbf{a}_{0} \in \mathcal{K}_{1}$ satisfying:

- $\chi_{1}\left(\mathbf{a}_{0}\right)=c_{\infty}$ (where $c_{\infty}$ is the parameter whose existence is guaranteed by Theorem 3);

- an iterate $f_{\mathbf{a}_{0}}^{k}\left(\omega_{2}\left(\mathbf{a}_{0}\right)\right)$ is equal to the fixed point of $f_{\mathbf{a}_{0}}$ corresponding to the $\beta$-fixed point of $z \mapsto z^{2}+c_{\infty}$;

- the set $\chi_{1}^{-1}\left(c_{\infty}\right)$ is a one-dimensional submanifold of $\mathcal{Q}_{1}$ locally at $\mathbf{a}_{0}$.

The first step in proving Theorem 7 is to establish that $\chi_{1}^{-1}\left(c_{\infty}\right)$ is an analytic set in $\mathcal{Q}_{1}$. The definition of an analytic set is as follows.

Definition 5. Suppose $D$ is an open set in $\mathbb{C}^{n}$. The set $A$ is called analytic in $D$ if for every $z \in D$ there exist an open neighborhood $U$ of $z$ in $D$ and a finite number of holomorphic functions $f_{i}: U \rightarrow \mathbb{C}, i=1, \ldots, k$, such that $A \cap U=\{z \in$ $\left.U \mid f_{1}(z)=\cdots=f_{k}(z)=0\right\}$. 
We will give a few properties of analytic sets. Proofs can be found in books on several complex variables, such as [KK] and [GF].

Let $\mathbf{a}_{0} \in A$. If there are functions $f_{1}, \ldots, f_{k}$, defined on a neighborhood $U$ of $\mathbf{a}_{0}$ such that $A \cap U=\left\{z \in U \mid f_{1}(z)=\cdots=f_{k}(z)=0\right\}$ and

$$
\operatorname{rank}\left(\frac{\partial f_{i}}{\partial z_{j}}\right)=k,
$$

then $\mathbf{a}_{0}$ is called a regular point or a manifold point. By the Implicit Function Theorem, $A$ is locally biholomorphically equivalent to a domain in $\mathbb{C}^{n-k}$ at $\mathbf{a}_{0}$. We define the dimension of $A$ at $\mathbf{a}_{0}$ to be $\operatorname{dim}_{\mathbf{a}_{0}} A=n-k$. A point in $A$ that is not regular is called singular, and we denote the singular points of $A$ by $S(A)$.

Proposition 8. Let $A$ be an analytic set in the open set $U \subset \mathbb{C}^{n}$. Then the set of singular points $S(A)$ is analytic in $U$, and the set of regular points $A \backslash S(A)$ is dense in $A$.

Clearly, every point is regular and of the same dimension in a neighborhood of a regular point. By the proposition, every singular point lies in the closure of the regular points. The following definition therefore makes sense:

Definition 6. The dimension of an analytic set $A \subset \mathbb{C}^{n}, A \neq \emptyset$, at $\mathbf{a}_{0}$ is

$$
\limsup _{\mathbf{a} \rightarrow \mathbf{a}_{0}} \operatorname{dim}_{\mathbf{a}}(A)
$$

where $\mathbf{a}$ tends to $\mathbf{a}_{0}$ through regular points. We define the dimension of $A$ by

$$
\operatorname{dim} A=\sup _{\mathbf{a} \in A} \operatorname{dim}_{\mathbf{a}} A .
$$

If $d=\operatorname{dim} A=\inf _{\mathbf{a} \in A} \operatorname{dim}_{\mathbf{a}} A$, we say that $A$ is purely $d$-dimensional.

Theorem 9. Suppose $A \neq \emptyset$ is analytic in the open set $U \subset \mathbb{C}^{n}$ and $S(A) \neq \emptyset$. Then

$$
\operatorname{dim} S(A) \leq \operatorname{dim} A-1 .
$$

An analytic set is called irreducible if it cannot be written as a union of two analytic sets $A=A_{1} \cup A_{2}$ with both $A_{1}$ and $A_{2}$ proper subsets of $A$. Every analytic set can be decomposed into irreducible components:

Theorem 10. Suppose $A$ is analytic in $U \subset \mathbb{C}^{n}$. Then $A$ has a unique representation $A=\bigcup_{\alpha} A_{\alpha}$ as a locally finite union of irreducible subsets, with $A_{\alpha} \not \subset A_{\beta}$ for $\alpha \neq \beta$. This decomposition is given by

$$
\left\{A_{\alpha}\right\}_{\alpha}=\{\bar{C} \mid C \text { is a connected component of } A \backslash S(A)\} .
$$

Here the closure $\bar{C}$ is taken in $U$.

Remark 1. It follows directly from Theorem 10 that if $A$ is analytic in $U \subset \mathbb{C}$ and of dimension 0 , then $A$ is discrete in $U$.

Most of the work to see that $\chi_{1}^{-1}\left(c_{\infty}\right)$ is analytic in $\mathcal{Q}_{1}$ has been carried out in [DH2]; in fact, they prove (Corollary 2, page 313) that for any analytic family $\left(f_{\lambda}\right)_{\lambda \in \Lambda}$ of quadratic-like mappings, and any parameter $c$ in the Mandelbrot set, the set of parameters $\lambda$ for which $f_{\lambda}$ is hybrid equivalent to $z^{2}+c$ is an analytic subset of $\Lambda$. The definition of an analytic family of polynomial-like maps is the following. 
Definition 7. Let $\Lambda$ denote a complex analytic manifold and suppose $\mathbf{f}=\left(f_{\lambda}\right.$ : $\left.U_{\lambda}^{\prime} \rightarrow U_{\lambda}\right)_{\lambda \in \Lambda}$ is a family of polynomial-like mappings. Set $\mathcal{U}^{\prime}=\{(\lambda, z) \mid \lambda \in \Lambda, z \in$ $\left.U_{\lambda}^{\prime}\right\}, \mathcal{U}=\left\{(\lambda, z) \mid \lambda \in \Lambda, z \in U_{\lambda}\right\}$ and $f(\lambda, z)=f_{\lambda}(z)$. We say that $\mathbf{f}$ is an analytic family of polynomial-like maps if the following three conditions are satisfied:

(1) $\mathcal{U}$ and $\mathcal{U}^{\prime}$ are homeomorphic over $\Lambda$ to $\Lambda \times \mathbb{D}$;

(2) the projection $\pi_{\Lambda}$ from the closure of $\mathcal{U}^{\prime}$ in $\mathcal{U}$ to $\Lambda$ is proper; and

(3) $f: \mathcal{U}^{\prime} \rightarrow \mathcal{U}$ is complex analytic and proper.

For parameters $\mathbf{a} \in \mathcal{Q}_{1}$, we have that $\omega_{1}$-renormalizations of $f_{\mathbf{a}}$ locally form an analytic family. More precisely:

Lemma 1. Given $\mathbf{a}_{0} \in \mathcal{Q}_{1}$, there are a bi-disk $\Lambda \subset \mathcal{Q}_{1}$ containing $\mathbf{a}_{0}$ and $\omega_{1}$ renormalizations $\left(f_{\mathbf{a}}: U_{\mathbf{a}}^{\prime} \rightarrow U_{\mathbf{a}}\right)_{\mathbf{a} \in \Lambda}$ that form an analytic family of quadratic-like mappings. In particular, $\chi_{1}^{-1}\left(c_{\infty}\right)$ is an analytic set in $\mathcal{Q}_{1}$.

Of course a similar statement about $\mathcal{Q}_{2}$ is true, and it follows that $\mathcal{Q}_{1}$ and $\mathcal{Q}_{2}$ are open sets.

Proof. Let $\mathbf{a}_{0} \in \mathcal{Q}_{1}$. Then there are topological disks $U, U^{\prime}$ with $\omega_{1}\left(\mathbf{a}_{0}\right) \in U^{\prime}$ such that $f_{\mathbf{a}_{0}}: U^{\prime} \rightarrow U$ is quadratic-like. Shrinking $U$ and taking for $U^{\prime}$ the component of $f_{\mathbf{a}_{0}}(U)$ that is compactly contained in $U$, we may assume that the boundaries of $U$ and $U^{\prime}$ are simple closed analytic curves and that the critical values $f_{\mathbf{a}_{0}}\left(\omega_{i}\left(\mathbf{a}_{0}\right)\right)$, $i=1,2$, are not contained in $\partial U$. Choose a bi-disk $\Lambda$ around $\mathbf{a}_{0}$ such that for all a in a neighborhood of $\bar{\Lambda}$, the critical values avoid $\partial U$. We then have a unique holomorphic motion $h: \Lambda \times \partial U^{\prime} \rightarrow \mathbb{C}$ of $\partial U^{\prime}$ such that $f_{\mathbf{a}}(h(\mathbf{a}, z))=f_{\mathbf{a}_{0}}(z) \in \partial U$. Since the image of a compact set under a holomorphic motion depends continuously on the parameter (in the Hausdorff metric on compact subsets of the sphere), we may suppose that $h_{\mathbf{a}}\left(\partial U^{\prime}\right)$ is contained in $U$ for all $\mathbf{a} \in \Lambda$, by shrinking $\Lambda$ if necessary. Note that $h(\mathbf{a}, \partial U)$ is a Jordan curve for each $\mathbf{a} \in \Lambda$, and denote by $U_{\mathbf{a}}^{\prime}$ the domain bounded by it. Then $f_{\mathbf{a}}: U_{\mathbf{a}}^{\prime} \rightarrow U$ is a quadratic-like mapping for each $\mathbf{a} \in \Lambda$. Set $\mathcal{U}^{\prime}=\left\{(\mathbf{a}, z) \mid \mathbf{a} \in \Lambda, z \in U_{\mathbf{a}}^{\prime}\right\}$ and $\mathcal{U}=\Lambda \times U$. We claim that $\left(f_{\mathbf{a}}: U_{\mathbf{a}}^{\prime} \rightarrow U_{\mathbf{a}}\right)_{\lambda \in \Lambda}$ is an analytic family of quadratic-like mappings. To see this we must verify (1)-(3) in the definition.

Let $\phi: U \rightarrow \mathbb{D}$ be a homeomorphism. Then $\Phi(\mathbf{a}, z)=(\mathbf{a}, \phi(z))$ is a homeomorphism over $\Lambda$ mapping $\mathcal{U}$ onto $\Lambda \times \mathbb{D}$. Let $\psi_{\mathbf{a}}: \mathbb{D} \rightarrow U_{\mathbf{a}}^{\prime}$ be the conformal map that takes 0 to $\omega_{1}(\mathbf{a})$ and whose derivative $\psi_{\mathbf{a}}^{\prime}(0)$ is real and positive. Then $\Psi(\mathbf{a}, z)=\left(\mathbf{a}, \psi_{\mathbf{a}}(z)\right)$ is a homeomorhism over $\Lambda$, mapping $\Lambda \times \mathbb{D}$ onto $\mathcal{U}^{\prime}$. This shows (1).

Note that the closure of $\mathcal{U}^{\prime}$ in $\mathcal{U}$ is $\left.\hat{\mathcal{U}}^{\prime}=(\mathbf{a}, z) \mid \mathbf{a} \in \Lambda, z \in \bar{U}_{\mathbf{a}}^{\prime}\right\}$. This follows since $\bar{U}_{\mathbf{a}}$ depends continuously on $\mathbf{a} \in \Lambda$ in the Hausdorff topology. Let $A \subset \Lambda$ denote a compact set. Then the preimage of this set under the projection of $\hat{\mathcal{U}}^{\prime}$ onto $\Lambda$ is $\left\{(\mathbf{a}, z) \mid \mathbf{a} \in A, z \in \bar{U}_{\mathbf{a}}^{\prime}\right\}$. Since $\bar{U}_{\mathbf{a}}^{\prime}$ depends continuously on $\mathbf{a}$, this set is compact. This shows (2).

Clearly $f:(\mathbf{a}, z) \mapsto f_{\mathbf{a}}(z)$ is complex analytic. Note that $f(\mathbf{a}, z)=f_{\mathbf{a}}(z)$ maps $\hat{\mathcal{U}}^{\prime} \backslash \mathcal{U}^{\prime}$ onto $\Lambda \times \partial U$. Hence the preimage $K^{\prime}$ of a compact set $K \subset \mathcal{U}$ avoids $\hat{\mathcal{U}}^{\prime} \backslash \mathcal{U}^{\prime}$. Using that $\pi_{\Lambda}\left(K^{\prime}\right)=\pi_{\Lambda}(K)$, it follows that $K^{\prime}$ does not intersect the boundary of $\mathcal{U}^{\prime}$. So $K^{\prime}$ is a closed and bounded subset of $\mathbb{C}^{2}$. We conclude that $f$ is proper, which finishes the proof of (3).

That $\chi_{1}^{-1}\left(c_{\infty}\right)$ is analytic in $\mathcal{Q}_{1}$ now follows directly by applying Corollary 2 on page 313 in [DH2] and Proposition [6] 

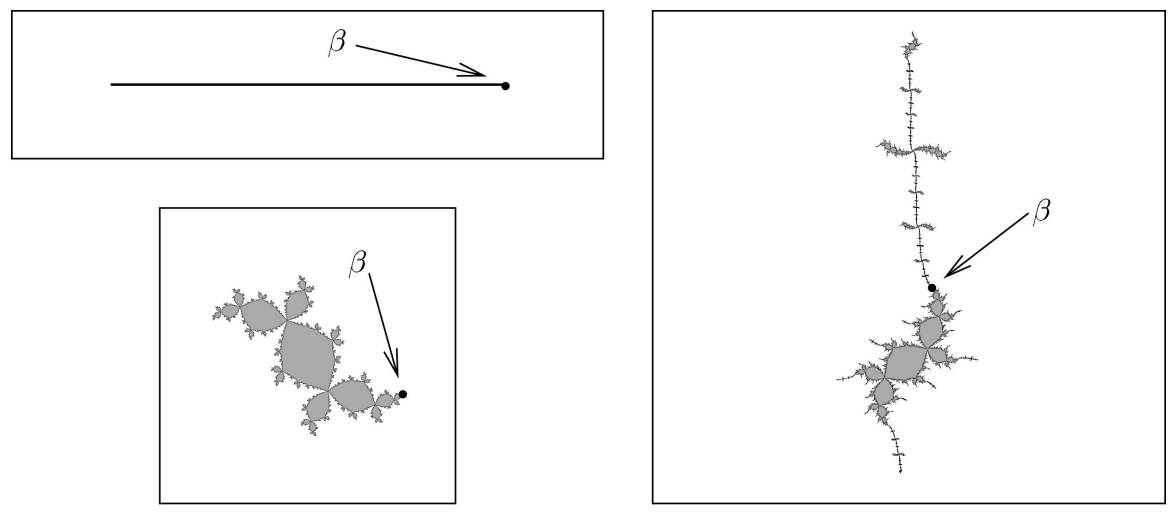

Figure 2. To the upper left is drawn an approximation of the Julia set of $z \mapsto z^{2}-2$. Below is the "rabbit", that is, the Julia set of $z \mapsto z^{2}+c$ with $c \approx-0.12236+0.74486 i$. To the right is illustrated the Julia set of $f_{\mathbf{a}}(z)=z^{3}-3 a^{2} z+b$, with $\mathbf{a}=(a, b) \approx(-0.03124478736-0.8615522432 i,-0.1514859674+$ $0.3334260962 i)$. This cubic polynomial is the result of intertwining the two mentioned quadratic polynomials at their $\beta$-fixed points. Notice the copies of the Julia sets of the quadratic maps inside the Julia set of the cubic polynomial.

Next we are going to state a result from $\mathrm{EY}$ (see also $\mathrm{Ha}$ ) that shows that Julia sets of quadratic polynomials show up inside Julia sets of certain cubic polynomials. To do so, we first need some notation. From the Straightening Theorem we know that for $\mathbf{a} \in \mathcal{Q}_{i}$, an $\omega_{i}$-renormalization has a unique fixed point corresponding to the $\beta$-fixed point. This point we denote by $\beta_{i}(\mathbf{a})$.

The following result is an immediate consequence of the work of Epstein and Yampolsky in $[\mathrm{EY}]$. They obtain it using intertwining surgery, although for this particular case the word "joining" surgery might be more appropriate since two quadratic-like mappings are joined together at their $\beta$-fixed points; see Figure 2

Theorem 11. There is a map $B: M \backslash\{1 / 4\} \times M \backslash\{1 / 4\} \rightarrow \mathcal{K}_{1} \cap \mathcal{K}_{2}$, which is a homeomorphism onto its image, mapping $\left(c_{1}, c_{2}\right)$ to $\mathbf{a}$, where $\mathbf{a}$ satisfies $\beta_{1}(\mathbf{a})=$ $\beta_{2}(\mathbf{a})$ and $\chi_{i}(\mathbf{a})=c_{i}, i=1,2$.

We can now finish the proof of Theorem 7

Let $\mathcal{Q}_{1}^{\prime}$ denote the component of $\mathcal{Q}_{1}$ that contains $B\left(\left\{c_{\infty}\right\} \times(M \backslash\{1 / 4\})\right)$. The set $A=\left(\left.\chi_{1}\right|_{\mathcal{Q}_{1}^{\prime}}\right)^{-1}\left(c_{\infty}\right)$ is analytic in $\mathcal{Q}_{1}^{\prime}$. This set cannot be of dimension 2 . If it were, then it would contain a bi-disk, which contradicts that $B:(M \backslash\{1 / 4\}) \times(M \backslash$ $\{1 / 4\}) \rightarrow \mathcal{Q}_{1}{ }^{\prime}$ is a homeomorphism. Since $A$ contains the homeomorphic image of $M \backslash\{1 / 4\}$, it follows that $A$ has dimension 1. In particular (see Remark 1 ), the set of singular points $S(A)$ is discrete in $\mathcal{Q}_{1}^{\prime}$.

Let $\mathcal{M}=\left\{c \in M \mid \exists k\left(P_{c}^{k}(0)=\beta(c)\right)\right\}$, where $\beta(c)$ denotes the $\beta$-fixed point of $P_{c}: z \mapsto z^{2}+c$. A standard normality argument (such as the one that shows that the boundary of $M$ is contained in the closure of the center parameter, see e.g. [CG]) shows that this set is dense in $\partial M$. Now, no point in $\mathcal{M} \subset M$ is isolated, and we deduce that infinitely many points in the image $B\left(\left\{c_{\infty}\right\}, \mathcal{M}\right)$ are regular. 
Taking $\mathbf{a}_{0}$ for a regular point in $B\left(\left\{c_{\infty}\right\} \times \mathcal{M}\right)$, the polynomial $f_{\mathbf{a}_{0}}$ has the required dynamical properties. This proves Theorem 7 .

Relating the dynamical plane to a parameter slice. Let $\mathbb{D} \rightarrow \mathbb{C}^{2}, t \mapsto \mathbf{a}(t)$, denote a parametrization of the complex submanifold given by Theorem 7 with $\mathbf{a}(0)=\mathbf{a}_{0}$. Abusing notation, we write $f_{t}=f_{\mathbf{a}(t)}, \beta_{1}(t)=\beta_{1}(\mathbf{a}(t))$ and $\omega_{i}(t)=$ $\omega_{i}(\mathbf{a}(t)), i=1,2$.

For all $t \in \mathbb{D}$, we have that $f_{t}$ is $\omega_{1}$-renormalizable and the renormalization is hybrid equivalent to $P_{c_{\infty}}$. We denote by $K^{1}(t)$ the filled Julia set of the $\omega_{1^{-}}$ renormalization of $f_{t}$. This set moves holomorphically with $t$ :

Theorem 12. There is a holomorphic motion $h: \mathbb{D} \times \mathbb{C} \rightarrow \mathbb{C}$ such that $h_{t}\left(K^{1}(0)\right)=$ $K^{1}(t)$ and $h_{t}$ commutes with the dynamics on $K^{1}(0)$, i.e.,

$$
h_{t} \circ f_{0}=f_{t} \circ h_{t}
$$

on $K^{1}(0)$.

Proof. Choose a period $p$ and denote by $Z_{p}(t)$ the set of points in $K^{1}(t)$ that are periodic of period $p$ under $f_{t}$.

Let $\mathcal{Y}_{p}=\left\{(t, z) \mid t \in \mathbb{D}, z \in Z_{p}(t)\right\}$, and let $\pi_{i}: \mathbb{C} \times \mathbb{C} \rightarrow \mathbb{C}$ denote the projections such that for every $\mathbf{a} \in \mathbb{C} \times \mathbb{C}$ we have that $\mathbf{a}=\left(\pi_{1}(\mathbf{a}), \pi_{2}(\mathbf{a})\right)$. We claim that the projection $\pi_{1}: \mathcal{Y}_{p} \rightarrow \mathbb{D},(t, z) \mapsto t$, is a covering map. Let $t_{0} \in \mathbb{D}$ denote an arbitrary point. Any $\omega_{1}$-renormalization $f_{t_{0}}: U^{\prime} \rightarrow U$ is hybrid equivalent to $P_{c_{\infty}}$; so $\# Z_{p}(t)$ is constant. The set $Z_{p}\left(t_{0}\right)$ contains no indifferent cycles, for the same reason. It then follows from the Implicit Function Theorem that there are a neighborhood $W$ of $t_{0}$ and holomorphic functions $z_{i}: W \rightarrow \mathbb{C}, i=1, \ldots, \# Z_{p}\left(t_{0}\right)$, such that $z_{i}(t) \in Z_{p}(t)$ for all $i$. Shrinking $W$, we can assume that $z_{i}-z_{j}$ is non-vanishing for $i \neq j$. That $\# Z_{p}(t)$ is constant implies that $\bigcup_{i}\left\{(t, z) \mid t \in W, z \in z_{i}(W)\right\}$ is an open neighborhood of the fiber $\pi_{1}^{-1}\left(t_{0}\right)$. The claim now follows because for each $i$ the map $\pi_{1}:\left\{(t, z) \mid t \in W, z \in z_{i}(W)\right\} \rightarrow W$ is a homeomorphism.

Using that the projection $\pi_{1}: \mathcal{Y}_{p} \rightarrow \mathbb{D}$ is a covering map, we can lift $\pi_{1}$ : $\mathbb{D} \times Z_{p}(0) \rightarrow \mathbb{D}$ to $\pi_{1}: \mathcal{X}_{p} \rightarrow \mathbb{D}$ and obtain a mapping $H: \mathbb{D} \times Z_{p}(0) \rightarrow \mathcal{Y}_{p}$.

Then define $h: \mathbb{D} \times Z_{p}(0) \rightarrow \mathbb{C}$ by $h=\pi_{2} \circ H$; i.e., such that the following diagram commutes:

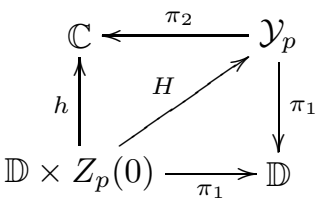

The map $h$ is a holomorphic motion: By the Implicit Function Theorem, $t \mapsto h(t, z)$ is holomorphic for each $z \in Z_{p}(0)$. Suppose $h_{t_{0}}(x)=h_{t_{0}}(y)$. Now $t \mapsto(t, h(t, x))$ is a lift of the identity to the map $\pi_{1}: \mathcal{Y}_{p} \rightarrow \mathbb{D}$, and the same is true for $t \mapsto(t, h(t, y))$. So by uniqueness of liftings, $h(t, x)=h(t, y)$ for all $t$. Since $z \mapsto h(0, z)$ is injective, it follows that $x=y$. Thus $z \mapsto h(t, z)$ is injective for all $t \in \mathbb{D}$.

The same line of arguments shows that $h$ commutes with the dynamics. Both the map $t \mapsto\left(t, f_{t} \circ h(t, x)\right)$ and $t \mapsto\left(t, h\left(t, f_{0}(x)\right)\right)$ are lifts of the identity map to $\pi_{1}: \mathcal{Y}_{p} \rightarrow \mathbb{D}$ that agree for $t=0$, and by uniqueness they agree for all $t$. Thus the equation

$$
h\left(t, f_{0}(x)\right)=f_{t} \circ h(t, x)
$$

holds for all $t \in \mathbb{D}$ and all $x \in Z_{p}(0)$. 
Noting that for each $t$ the sets $Z_{1}(t), Z_{2}(t), \ldots$ are disjoint, we get a holomorphic motion $h: \mathbb{D} \times \bigcup_{p} Z_{p}(0)$ that commutes with the dynamics.

By the Straightening Theorem, $\bigcup_{p} Z_{p}(0)$ is dense in $K^{1}(0)$, so by the $\lambda$-lemma (see [MSS]) we can extend $h$ continuously to a holomorphic motion $h: \mathbb{D} \times K^{1}(0) \rightarrow$ $\mathbb{C}$. Now $h\left(t, K^{1}(0)\right)=K^{1}(t)$ by continuity; and, also by continuity, the extension commutes with the dynamics. $\mathbb{C}$.

Finally, using Słodkowski's Theorem (see $[\mathrm{Sl}]$ ), we can extend $h$ to $h: \mathbb{D} \times \mathbb{C} \rightarrow$

Define the connectedness locus $\mathcal{S}$ of the family $\left\{f_{t}\right\}_{t \in \mathbb{D}}$ by

$$
\mathcal{S}=\left\{t \in \mathbb{D} \mid K\left(f_{t}\right) \text { is connected }\right\} .
$$

Remark 2. Using a theorem of McMullen (see [Mc3]), it can be shown that $\mathcal{S}$ contains a quasiconformal image of the connectedness locus $M_{d} \subset \mathbb{C}$ of the family $\left\{z \mapsto z^{d}+c\right\}_{c \in \mathbb{C}}$, with the image of the boundary of $M_{d}$ contained in the boundary of $\mathcal{S}$, for some $d \geq 2$.

We will not use or prove this fact; instead we will show that the connectedness locus also contains a copy of $J_{\infty}$. For this we need a map relating the parameter disk $\{t \in \mathbb{D}\}$ to the dynamical plane $\{z \in \mathbb{C}\}$ of $f_{0}$.

Lemma 2. Let $h_{t}(z)=h(t, z)$, and denote by $k$ the preperiod of $\omega_{2}(0)$ under $f_{0}$. Then the mapping $H: \mathbb{D} \rightarrow \mathbb{C}, t \mapsto h_{t}^{-1} \circ f_{t}^{k}\left(\omega_{2}(t)\right)$, is quasiregular on domains compactly contained in $\mathbb{D}$ and non-constant. In fact, $H$ is $K$-quasiregular on $\{t<$ $\rho\}, \rho<1$, with $K=(1+\rho) /(1-\rho)$.

Proof. Let us first show that $H$ is not constant. If it were, then for all $t \in \mathbb{D}$ we would have $f_{t}^{k}\left(\omega_{2}(t)\right)=h(\beta(0))=\beta(t)$, where the last equality is due to the fact that $h$ commutes with the dynamics. However, by Theorem 11 we can find a sequence of parameters $t_{n}$, converging to 0 , such that $f_{t_{n}}$ is $\omega_{2}$-renormalizable and the critical point of the renormalization is not prefixed.

Let us now show that $H$ is quasiregular on domains compactly contained in $\mathbb{D}$. To see this, we will use the fact that the time $t$ map of a holomorphic motion is quasiconformal. Indeed, it follows from Proposition 5 of $[\mathrm{D}]$ (compare with the $\lambda$-lemma in $[\mathrm{MSS}]$ ) that for $|t|<\rho<1$ the mapping $h_{t}$ is $K$-quasiconformal with $K=(1+\rho) /(1-\rho)$.

Taking the $\frac{\partial}{\partial t}$ distributional derivate of the equation

$$
h_{t} \circ H(t)=f_{t}^{k}\left(\omega_{2}(t)\right)
$$

we get

$$
\left.\frac{\partial}{\partial \bar{t}} h_{t}\right|_{H(t)}+\left.\frac{\partial}{\partial z} h_{t}\right|_{H(t)} \frac{\partial H}{\partial \bar{t}}(t)+\left.\frac{\partial}{\partial \bar{z}} h_{t}\right|_{H(t)} \frac{\partial \bar{H}}{\partial \bar{t}}=\frac{\partial}{\partial \bar{t}} f_{t}^{k}\left(\omega_{2}(t)\right) .
$$

Since $t \mapsto h(t, z)$ and $t \mapsto f_{t}^{k}\left(\omega_{2}(t)\right)$ are holomorphic functions, it follows that

$$
\left.\frac{\partial}{\partial z} h_{t}\right|_{H(t)} \frac{\partial H}{\partial \bar{t}}(t)+\left.\frac{\partial}{\partial \bar{z}} h_{t}\right|_{H(t)} \frac{\partial \bar{H}}{\partial \bar{t}}=0 .
$$

Rearranging and taking absolute values, we obtain

$$
\left|\frac{\partial H / \partial \bar{t}}{\partial H / \partial t}(t)\right|=\left|\frac{\partial h_{t} / \partial \bar{z}}{\partial h_{t} / \partial z}(H(t))\right| .
$$

So $H$ is $K$ quasiregular on $\{|t|<\rho\}$, for $\rho<1$, with $K=(1+\rho) /(1-\rho)$. 
Main theorem. We can now prove the main theorem:

Main Theorem. There exist two cubic polynomials $f, g$ which have no indifferent cycles and which are not topologically conjugate on their Julia sets, but which nevertheless have the same rational lamination. In particular, $f$ and $g$ are not combinatorially rigid.

Proof. We denote by $\psi: U^{\prime} \rightarrow V^{\prime}$ a hybrid equivalence that maps a neighborhood $U^{\prime}$ of $K^{1}(0)$ to a neighborhood $V^{\prime}$ of $K\left(P_{c_{\infty}}\right)$ and conjugates $f_{0}: U^{\prime} \rightarrow U$ to $P_{c_{\infty}}$. Using that $\psi$ is a conjugacy and Proposition 5 , we get that $\beta_{1}(0)$ is accumulated by arbitrary small copies of $\hat{J}_{\infty}=\psi^{-1}\left(J_{\infty}\right)$, each of which is being mapped bijectively to $\hat{J}_{\infty}$ by an iterate of $f_{0}$. Since $H$ is quasiregular and not constant, it maps 0 to $\beta_{1}(0)$ with a local degree $d>0$. The copies of $\hat{J}_{\infty}$ are full and do not contain $\beta_{1}(0)$, and from this it follows that we can find a copy $\hat{J}_{\infty}^{\prime}$ and an integer $l>0$ such that

- $f_{0}^{l}$ maps $\hat{J}_{\infty}^{\prime}$ bijectively onto $\hat{J}_{\infty}$; and

- $H^{-1}\left(\hat{J}_{\infty}^{\prime}\right)$ intersected with a neighborhood of 0 is the disjoint union of $d$ continua $C_{1}, \ldots, C_{d}$ such that $H$ maps each $C_{i}$ bijectively onto $\hat{J}_{\infty}^{\prime}$.

By bijectivity there is exactly one point $t_{1} \in C_{1}$ such that $P_{c_{\infty}}^{l} \circ \psi\left(t_{1}\right)=0 \in J_{\infty}$. Since $h$ commutes with the dynamics on $K^{1}(0)$, we get

$$
f_{t_{1}}^{k+l}\left(\omega_{2}\left(t_{1}\right)\right)=\omega_{1}\left(t_{1}\right) .
$$

By injectivity of $\left.H\right|_{C_{1}}$, we get that if $t_{2} \in C_{1}$ and $t_{2} \neq t_{1}$, then $f_{t_{1}}^{k+1}\left(\omega_{2}\left(t_{1}\right)\right) \neq$ $\omega_{1}\left(t_{1}\right)$.

We claim that $f=f_{t_{1}}$ and $g=f_{t_{2}}$ for $t_{2} \in C_{1} \backslash\left\{t_{1}\right\}$ will satisfy the requirements of the theorem. We must prove:

(a) $f$ and $g$ do not possess indifferent cycles;

(b) $f$ and $g$ are not topologically conjugate on their Julia sets; and

(c) $f$ and $g$ have the same rational lamination, $\lambda_{\mathbb{Q}}(f)=\lambda_{\mathbb{Q}}(g)$.

Part (a) is an immediate consequence of the following lemma:

Lemma 3. Suppose $t \in \mathbb{D}$. If there exists an integer $k>0$ such that $f_{t}^{k}\left(\omega_{2}(t)\right) \in$ $K^{1}(t)$, then

(1) the filled Julia set of $f_{t}$ has no interior: $K\left(f_{t}\right)=J\left(f_{t}\right)$, and

(2) all cycles of $f_{t}$ are repelling.

Part (b). Suppose there exists a homeomorphism $\eta: J(f) \rightarrow J(g)$ that conjugates $f$ to $g$ on $J(f)$. By Lemma $3 K(f)=J(f)$ and $K(g)=J(g)$. The critical points $\omega_{i}\left(t_{1}\right)$ are distinguished in $K(f)$ in that they are the only points that have only one preimage. The critical points $\omega_{i}\left(t_{2}\right)$ are likewise distinguished in $K(g)$. It follows, since $\eta$ is a conjugacy, that $\eta$ maps the two critical points of $f$ onto the two critical points of $g$. By equation (1), we must have either $g^{k+l}\left(\omega_{2}\left(t_{2}\right)\right)=\omega_{1}\left(t_{2}\right)$ or $g^{k+l}\left(\omega_{1}\left(t_{2}\right)\right)=\omega_{2}\left(t_{2}\right)$. However, we have already seen that $g^{k+l}\left(\omega_{2}\left(t_{2}\right)\right) \neq \omega_{1}\left(t_{2}\right)$, and we cannot have $g^{k+l}\left(\omega_{1}\left(t_{2}\right)\right)=\omega_{2}\left(t_{2}\right)$, since all the forward images of $\omega_{1}\left(t_{2}\right)$ are contained in $K^{1}\left(t_{2}\right)$ and $\omega_{2}\left(t_{2}\right)$ is not an element in $K^{1}\left(t_{2}\right)$. This proves (b).

Part (c). To complete the proof we shall show that the polynomials $f_{t}$ have the same rational lamination for all $t \in C_{1}$. We will show that every rational ray lands, and that the landing point depends holomorphically on $t$ for values of $t$ in a neighborhood of $C_{1}$. This will follow from the following stability result (Proposition 2 of exposé 8 in the Orsay Notes, [DH1]). 
Proposition 13. Let $P_{0} \in \mathcal{P}_{d}$ and $\theta \in \mathbb{Q} / \mathbb{Z}$. Assume that $R_{P_{0}}(\theta)$ lands at the (pre)periodic point $\gamma_{0} \in J\left(P_{0}\right)$ and that the corresponding cycle is repelling. If $P_{0}^{i}\left(\gamma_{0}\right)$ is not a critical point for any $i \geq 0$, then there is a neighborhood $\Lambda$ of $P_{0}$ in $\mathcal{P}_{d}$ such that

(1) the ray $R_{P}(\theta)$ lands at a (pre)periodic repelling point $\gamma(P)$ for all $P \in \Lambda$;

(2) the map $\Lambda \times\{\eta \geq 0\} \rightarrow \mathbb{C}$ that maps $(P, \eta)$ to the point of potential $\eta$ on the ray $R_{P}(\theta)$ is continuous and holomorphic in $P$.

Indeed, fix a rational angle $\theta$ and a parameter $t_{0} \in C_{1}$. By Proposition 1 the dynamical ray of angle $\theta$ lands at a (pre)periodic point that is either parabolic or repelling. We have seen that $f_{t}$ has no indifferent cycles. So to apply Proposition 13 we just have to see that the (forward) critical orbits do not contain the landing point. This follows easily, since the forward orbits of the critical point $\omega_{i}\left(t_{0}\right)$ are trapped in a forward invariant region on which $f_{t_{1}}$ is conjugate to $P_{c_{\infty}}: J_{\infty} \rightarrow J_{\infty}$. By Theorem 4, $J_{\infty}$ contains no (pre)periodic points. So the critical orbits do not contain any (pre)periodic point such as the landing point of $R_{t_{0}}(\theta)$.

We conclude that the ray $R_{t}(\theta)$ keeps landing and moves holomorphically in a neighborhood of each point in $C_{1}$.

Suppose that the rays of angles $\theta_{1}$ and $\theta_{2}$ land at distinct points, for a parameter $t_{0} \in \mathbb{D}$. Because the landing points depend holomorphically on $t$ in a neighborhood of $t_{0}$, they will keep on landing at distinct points for all parameters in a neighborhood of $t_{0}$. Conversely, suppose two rational rays land at the same point for a parameter $t_{0} \in C_{1}$. Since the rays are disjoint and the rays including landing points depend holomorphically on $t$ in a neighborhood of $t_{0}$, we get from Hurwitz's Theorem that the two rays keep landing at the same point for all parameters in a neighborhood of $t_{0}$. Noting that $C_{1}$ is connected, we conclude that if two rays land at the same point for a parameter $t_{0} \in C_{1}$, they will do so for all parameters $t \in C_{1}$. So $\lambda_{\mathbb{Q}}\left(f_{t}\right)=\lambda_{\mathbb{Q}}\left(f_{0}\right)$ for all $t \in C_{1}$.

We still have to prove Lemma 3. To do so we will use the fact that the multiplier at an indifferent fixed point is a quasiconformal invariant.

Lemma 4. Suppose the two holomorphic germs $g_{1}, g_{2}:(\mathbb{C}, 0) \rightarrow(\mathbb{C}, 0)$ have indifferent fixed points at the origin, and a quasiconformal germ conjugates $g_{1}$ to $g_{2}$. Then the multiplier $g_{1}^{\prime}(0)$ is equal to the multiplier $g_{2}^{\prime}(0)$.

The lemma is well known; in fact, Naisshul' Nai] proves that the multiplier of an indifferent fixed point is even a topological invariant.

We now prove Lemma 3 ,

Proof. It follows from the classification of Fatou components that (2) implies (1); so we need only prove (2). First note that $K^{1}(t)$ does not contain any non-repelling cycles. Indeed, the $\omega_{1}$-renormalization of $f_{t}$ is hybrid equivalent to $P_{c_{\infty}}$. So if $K^{1}(t)$ contains an indifferent cycle, then also $P_{c_{\infty}}$ possesses such a cycle by Lemma 4 But Theorem 4 asserts that all the cycles of $P_{c_{\infty}}$ are repelling.

We distinguish two cases, supposing $f_{t}$ has a non-repelling cycle.

Case 1: The cycle is contained in $J\left(f_{t}\right)$. Then it is indifferent. Since both critical orbits are captured by $K^{1}(t)$, in the sense that $f_{t}^{n}\left(\omega_{1}(t)\right) \in K^{1}(t)$ for all $n \geq 0$ and $f_{t}^{n}\left(\omega_{2}(t)\right) \in K^{1}(t)$ for all $n \geq k$, the cycle must be contained in $K^{1}(t)$. We have seen that this cannot be the case. 
Case 2: The cycle is contained in the interior of $K\left(f_{t}\right)$. Again, since the orbits of the critical points are captured by $K^{1}(t)$ and (by Theorem 4) this set has no interior, the cycle cannot be attracting. By the classification of Fatou components, it is necessarily a Siegel cycle. The boundary of the cycle of Siegel disks is contained in the closure of the postcritical set, and thus in $K^{1}(t)$. Since $K^{1}(t)$ is a full set, the Siegel disks themselves are also contained in $K^{1}(t)$. As before, this is not the case.

Question. I pass on a question I was asked by McMullen during a presentation of the result of this paper.

Question 1. Is there a natural combinatorial object associated to a polynomial $f \in \mathcal{C}_{d}, d>2$, that uniquely determines the dynamics of $f$ on $J(f)$ ?

\section{ACKNOWLEDGMENT}

I thank Bodil Branner and Carsten Petersen for reading and commenting on a version of this paper. Likewise I am grateful to Xavier Buff and Adrian Douady for instructive discussions.

\section{REFERENCES}

[BH1] B. Branner and J. Hubbard, The iteration of cubic polynomials, Part I: The global topology of parameter space, Acta Math., 160 (1988), no 3-4, 143-206. MR 90d:30073

[CG] L. Carleson and T. Gamelin, Complex Dynamics, Springer-Verlag, (1993). MR 94h:30033

[D] A. DouADY, Prolongement de mouvements holomorphes (d'après Stodkowski et autres), (French) [Extension of holomorphic motions (after Slodkowski and others)] Séminaire Bourbaki, Vol. 1993/94. Astérisque No. 227 (1995), Exp. No. 775, 3, 7-20. MR 95m:58104

[DH1] A. Douady and J. H. Hubbard, Etude dynamique des polynômes complexes I and II, Publ. Math. d'Orsay (1984-85). MR 87f:58072a

[DH2] A. Douady and J. HubBard, On the dynamics of polynomial-like mappings, Ann. Sci. École Norm. Sup. Paris (4) 18 (1985), 287-343. MR 87f:58083

[EY] A. L. Epstein and M. YAmpolsky, Geography of the Cubic Connectedness Locus: Intertwining Surgery, Ann. Sci. École Norm. Sup. Paris (4), 32 (1999), no. 2, 151-185. MR 2000i:37067

[GF] H. Grauert and K. Fritzsche, Several Complex Variables, Springer-Verlag, (1976). MR 54:3004

[Ha] P. Hä̈ssinsky, Thèse de doctorat, Université Paris-Sud in Orsay, (1998).

[KK] L. KAUP and B. KAUP, Holomorphic Functions of Several Variables, Walter de Gruyter, (1983). MR 85k:32001

[L1] M. LYUBICH, The quadratic family as a qualitatively solvable model of chaos, Notices Amer. Math. Soc. 47 (2000), no. 9, 1042-1052. MR 2001g:37063

[L2] M. Lyubich, Dynamics of quadratic polynomials. I, II. Acta Math. 178 (1997), no. 2, 185-247, 247-297. MR 98e:58145

[MSS] R. MaÑÉ, P. SAD and D. P. Sullivan, On the dynamics of rational maps, Ann. Sci. École Norm. Sup. Paris (4) 16 (1983), no. 2, 193-217. MR 85j:58089

[M1] J. Milnor, Dynamics in one complex variable, Introductory Lectures, Vieweg, 1999. MR 2002i:37057

[M2] J. Milnor, Local connectivity of Julia sets: Expository Lectures, The Mandelbrot Set, Theme and Variations, edited by Tan Lei, Cambridge University Press (2000), pp. 67-116. MR 2001b:37073

[Mc1] C. T. MCMullen, The Classification of Conformal Dynamical Systems, Current Developments in Mathematics, 1995 (Cambridge, MA), 323-360, Internat. Press, Cambridge, MA, (1994). MR 98h:58162

[Mc2] C. T. McMullen, Complex Dynamics and Renormalization, Annals of Mathematical Studies 135, Princeton University Press, (1994). MR 96b:58097 
[Mc3] C. T. McMullen, The Mandelbrot Set is Universal, The Mandelbrot Set: Theme and Variations, edited by Tan Lei, (2000), pp. 1-17. MR 2002f:37071

[Nai] V. A. NAǏsHUL', Topological invariants of analytic and area preserving mappings and their applications to analytic differential equations in $\mathbb{C}^{2}$ and $\mathbb{C P}^{2}$, Trans. Moscow Math. Soc. 42 (1983), 239-250. MR 84f:58092

[Sch] D. SchleICher, On fibers and local connectivity of Mandelbrot and Multibrot Sets. Manuscript (1998).

[Sl] Z. SŁOdKOwski, Extensions of holomorphic motions, Ann. Scuola Norm. Sup. Pisa Cl. Sci. (4) 22 (1995), 185-210. MR 96k:30026

$[\mathrm{S} \varnothing] \quad$ D. E. K. SøRENSEn, Infinitely renormalizable quadratic polynomials, with non-locally connected Julia set. J. Geom. Anal. 10 (2000), no 1, 169-206. MR 2001e:37057]

Université Paul Sabatier, Laboratoire Emile Picard, 118, route de Narbonne, 31062 Toulouse Cedex, France

E-mail address: chris@picard.ups-tlse.fr

Current address: Department of Mathematics, Technical University of Denmark, Matematiktorvet, building 303, DK - 2800 Kgs Lyngby, Denmark

E-mail address: christian.henriksen@mat.dtu.dk 